\title{
Lateral First Obtuse Marginal Branch Artery
}

National Cancer Institute

\section{Source}

National Cancer Institute. Lateral First Obtuse Marginal Branch Artery. NCl Thesaurus.

Code C102308.

The lateral branch distal to a bifurcation of the first obtuse marginal artery. 